\title{
Integrative analysis of 5-methyl- and 5-hydroxymethylcytosine indicates a role for 5-hydroxymethylcytosine as a repressive epigenetic mark
}

Christopher E. Schlosberg ${ }^{1}$, John R. Edwards ${ }^{1, *}$

${ }^{1}$ Center for Pharmacogenomics, Department of Medicine, Washington University in St. Louis School of Medicine, St. Louis, MO, USA.

* To whom correspondence should be addressed. Tel: 314-362-6935; Email: jredwards@wustl.edu 


\section{ABSTRACT}

Since the discovery of 5 -hydroxymethylcytosine $(5 \mathrm{hmC})$ as a prominent DNA base modification found in mammalian genomes, an emergent question has been what role this mark plays in gene regulation. $5 \mathrm{hmC}$ is hypothesized to function as an intermediate in the demethylation of 5-methylcytosine $(5 \mathrm{mC})$ and also in reactivation of silenced regulatory elements, including promoters and enhancers. Further, weak positive correlations have been observed between gene body $5 \mathrm{hmC}$ and gene expression. We previously demonstrated that ME-Class, which uses a high-resolution model of whole-genome bisulfite sequencing data, is an effective tool to understand relationships between $5 \mathrm{mC}$ and expression. In this work, we present ME-Class2, a machine-learning based tool to perform integrative 5mCG, 5hmCG and expression analysis. Using ME-Class2 we analyze whole-genome single-base resolution $5 \mathrm{mC}$ and $5 \mathrm{hmC}$ datasets from 20 primary tissue and cell samples to uncover relationships between $5 \mathrm{hmC}$ and expression. The addition of $5 \mathrm{hmC}$ improves model performance for tissues with high-levels of $5 \mathrm{hmC}$ such as the brain. Our analysis further indicates that conversion of $5 \mathrm{mC}$ to $5 \mathrm{hmC}$ within $2 \mathrm{~kb}$ of the transcription start site associates with distinct functions depending on the summed level of $5 \mathrm{mC}+5 \mathrm{hmC}$. Unchanged levels of $5 \mathrm{mC}+5 \mathrm{hmC}$ (i.e. conversion from $5 \mathrm{mC}$ to stable $5 \mathrm{hmC}$ ) associate with repression. Meanwhile, decreases in $5 \mathrm{mC}+5 \mathrm{hmC}$ (i.e. $5 \mathrm{hmC}$-mediated demethylation) associate with gene activation. As more large-scale, genome-wide, differential DNA methylation studies become available, tools such as MEClass2 will prove invaluable to interpret epigenomic data and guide mechanistic studies into the function of $5 \mathrm{hmC}$. 


\section{INTRODUCTION}

In mammalian genomes, cytosines are frequently covalently modified at the 5-position with methyl-, hydroxymethyl-, formyl-, and carboxy- groups. The initial modification occurs by addition of a methylgroup to the 5-position of the cytosine (5-methylcytosine, $5 \mathrm{mC}$ ) by a DNA methyltransferase (Dnmt). The subsequent modifications are then formed through successive oxidation of $5 \mathrm{mC}$ by the Ten-eleven translocation (Tet) family of enzymes. While $5 \mathrm{mC}$ occurs at nearly $70 \%$ of all CG dinucleotides (CpG) in the genome in all tissues, 5-hydroxymethylcytosine $(5 \mathrm{hmC})$ appears to be primarily limited to embryonic stem cells, neurons, liver, breast, testis, and placenta tissues, occurring at 2-17\% of CpGs depending on the tissue type (1-3). Meanwhile 5hmC's oxidized derivatives, 5-formylcytosine (5fC) and 5carboxycytosine ( $5 \mathrm{caC})$, are only found at very low levels, $10-1000$ fold less than $5 \mathrm{hmC}(1)$. It is still unclear whether $5 \mathrm{fC}$ and $5 \mathrm{caC}$ are short-lived intermediates (2) or whether they have an independent biological function in vivo $(3,4)$. Of these marks, $5 \mathrm{mC}$ has been the most studied and is a wellestablished player in maintaining inactivation of the silenced $X$ chromosome, mono-allelic gene expression at imprinted loci, and silencing retrotransposons (5). Abnormal patterns of $5 \mathrm{mC}$ are also linked to transcriptional dysregulation in cancer.

At the biochemical level, $5 \mathrm{hmC}$ likely plays a role in demethylation through both passive and active mechanisms. While Dnmt1 is responsible for copying and propagating $5 \mathrm{mC}$ during cell division, no similar mechanism has yet been discovered for $5 \mathrm{hmC}$. Notably, $5 \mathrm{hmC}, 5 \mathrm{fC}$, and $5 \mathrm{caC}$ are found at their highest levels in post-mitotic cells and are passively diluted during cell division. Active demethylation occurs through conversion of $5 \mathrm{mC}$ to $5 \mathrm{caC}$ via $5 \mathrm{hmC}$ and $5 \mathrm{fC}$ intermediates. $5 \mathrm{caC}$ is then converted to unmethylated cytosine through base excision repair or decarboxylation (6). In support of 5hmC's role as an intermediate in demethylation in vivo, Tet2-/- mouse brains exhibit low level gains in methylation (7).

What is known about the biological roles of $5 \mathrm{hmC}$ and the Tet family of enzymes (Tet1, Tet2, Tet3) are mostly supported through a combination of genetic and correlative studies. Genomic analyses show that while the majority of the genome is marked by $5 \mathrm{mC}$ except for $\mathrm{CpG}$ islands (CGIs), gene promoters, and enhancers, $5 \mathrm{hmC}$ is enriched at enhancers, gene bodies, and CGI shores. Further, $5 \mathrm{hmC}$ is depleted from CGIs in ES cells and neurons and depleted from intergenic regions in ES cells (4-6). $5 \mathrm{hmC}$ starts at low levels in the developing brain, but accumulates in the adult brain (8). Tet1-/- mice do not show impaired differentiation $(1,9)$ and have normal brain morphology $(10)$. However, they do show impairments in synaptic plasticity and memory extinction (10). Tet1-, Tet2-, Tet3-triple KO mice displayed impaired differentiation and embryonic development, and significant promoter hypermethylation (9). These data suggest compensatory effects between Tet family members. $5 \mathrm{hmC}$ may also play a role in enhancer regulation, as Tet2 deletion causes an increase in enhancer $5 \mathrm{mC}$ levels and reduced enhancer activity (11).

There has been some evidence that $5 \mathrm{hmC}$ can play a regulatory role independent of its role as an intermediate in demethylation in post-mitotic neurons and ES cells. For example, MeCP2 displays reduced affinity for hmCG compared to $\mathrm{mCG}$, and therefore conversion of $\mathrm{mCG}$ to stable $\mathrm{hmCG}$ in the 
neuronal genome may lead to loss of functional binding sites for MeCP2 (11-13). However, screens for $5 \mathrm{hmC}$ interacting factors have uncovered few $5 \mathrm{hmC}$-specific interactors (4). In general, $5 \mathrm{hmC}$ in gene bodies is frequently associated with gene expression; this includes both as a stable mark in neurons, and as an intermediate for demethylation. More recent studies in human liver and lung tissues observed $5 \mathrm{hmC}$ as a marker of active transcription associated with H3K4me1 at CpG island shores (12). However, whether $5 \mathrm{hmC}$ plays a role in promoter regulation is still unclear, and how $5 \mathrm{mC}$ and $5 \mathrm{hmC}$ signals in promoters and gene bodies synergize to affect gene silencing has not been studied.

We previously developed ME-Class to model methylation both at the gene promoters and in gene bodies to identify genes with a high probability of association between $5 \mathrm{mC}$ and gene expression (13). Here, we extend its functionality to incorporate $5 \mathrm{hmC}$ to systematically interrogate how changes in $5 \mathrm{mC}$ and $5 \mathrm{hmC}$ associate with gene expression. Our results indicate that models that include both $5 \mathrm{mC}$ and $5 \mathrm{hmC}$ out-perform $5 \mathrm{mC}$ only models, but only in tissues or cells (such as neuronal tissues) that have high levels of $5 \mathrm{hmC}$. Further, our results indicate that $5 \mathrm{hmC}$ associates with gene activation when it is involved in demethylation and with gene repression when it is stably present at and around the promoter of a gene.

\section{MATERIALS AND METHODS}

\section{WGBS, TAB-seq, oxBS-seq, and RNA-seq data}

Mapped sequence reads for whole genome bisulfite sequencing (WGBS), Tet-assisted bisulfite sequencing (TAB-seq), and RNA-seq in liver and lung tumor and matched normal samples were obtained from $\mathrm{Li}$ et al. (12) and from dendritic cells from Pacis et al. (14). WGBS, oxidative bisulfite sequencing (oxBS-seq), and RNA-seq from fetal and 6 week mouse brain samples were obtained from Lister et al. (7) and granule cells from Mellen et al. (15). WGBS and TAB-seq for human cortex are from Wen et al. (16). Corresponding RNA-seq data are from Brawand et al. (17) as used by Wen et al. (16).

\section{Estimation of $5 \mathrm{mC}$ and $5 \mathrm{hmC}$ levels}

$5 \mathrm{mC}$ and $5 \mathrm{hmC}$ levels were estimated using maximum likelihood methylation levels (MLML) from either TAB-seq or oxBS-seq (18). MLML provides a simultaneous maximum likelihood based on binomial estimates of $5 \mathrm{hmC}$ and $5 \mathrm{mC}$. We used MLML with a significance level of $\alpha=0.05$ for the binomial test at each CpG site and an expectation maximization convergence threshold of $1 \mathrm{e}-10$. Counts of individual CpGs with estimated $5 \mathrm{hmC}$ and $5 \mathrm{mC}$ in all samples can be found in Supplementary Table $\mathrm{S} 1$.

\section{Differential Expression from RNA-seq}

RNA-seq data from human liver, lung, and cortex samples were mapped to hg19 using HISAT2 (19). We used featureCounts to estimate feature counts over RefSeq reads (20). Differentially expressed genes were defined as abs[fold change] $>=2$ after applying a floor of $\mathrm{cpm}=1$. To create a standardized gene set with high quality methylation data, we excluded genes with ambiguous or incomplete transcription start 
site (TSS) annotations, genes shorter than $5 \mathrm{~kb}$, genes with $<40 \mathrm{CpGs}$ assayed within $+/-5 \mathrm{~kb}$ of the TSS, genes where, for all CpGs within $+/-5 \mathrm{~kb}$ of the TSS, the change in methylation (mCG/CG) was less than 0.2 , and genes with alternative promoters. These filters were used to exclude non-coding genes, pseudogenes, genes shorter than the interpolation boundary (see HRPS model description below), genes with low numbers of $\mathrm{CpGs}$ (to reduce bias caused by error in individual $\mathrm{CpG}$ measurements), and genes with no methylation changes at their respective promoters. We only included RefSeq genes with cdsStartStat and cdsEndStat flags marked as 'cmpl' according to the UCSC Table Browser. For any RefSeq genes with multiple RefSeq IDs corresponding to the same TSS location, we used a single RefSeq ID with the lowest accession number and excluded the remainder. This is a conservative method to simplify the annotations of genes with alternative promoter annotations. A full summary of differentially expressed filtered gene counts can be found in Supplementary Table S2.

\section{$5 \mathrm{hmC}$ incorporation in ME-Class}

MLML produces an estimate of $5 \mathrm{mC}$ and $5 \mathrm{hmC}$ for each $\mathrm{CpG}$ site. ME-Class high-resolution promoter signature (HRPS), region of interest (ROI), and whole-scale gene (WSG) models described in Schlosberg et al. (13) were extended to add 5hmCG features (Fig. 1a,b). For the HRPS model, 5mCG and 5hmCG data were independently interpolated using PCHIP interpolation and Gaussian smoothing (50bp bandwidth) across the window $+/-5 \mathrm{~kb}$ relative to each gene's TSS. Interpolated curves for $\Delta 5 \mathrm{hmCG} / \mathrm{CG}$ and $\triangle 5 \mathrm{mCG} / \mathrm{CG}$ (i.e. the difference in $5 \mathrm{hmCG}$ and $\mathrm{mCG}$ levels between samples) were discretized to create feature vectors for classification using the average methylation in each 20bp segment. Bins for the ROI model were inspired by Lou et al. (21). Differential $5 \mathrm{mCG}$ and $5 \mathrm{hmCG}$ levels were computed for each bin, which was then used in the resultant feature vector. For the WSG model, 5mCG and 5hmCG data were scaled to a constant length between the TSS and RefSeq annotated transcription end site (TES). Feature vectors were created from 125 bins upstream of the gene, 125 bins downstream of the gene, and 500 bins from the area between the TSS and TES. Differential $5 \mathrm{mCG}$ and $5 \mathrm{hmCG}$ levels were both computed for the entire set of bins and then combined to form the final feature vector. $\Delta 5 \mathrm{mCG} / \mathrm{CG}$ corresponds to the $5 \mathrm{mC}$ feature vector and $\triangle 5 \mathrm{hmCG} / \mathrm{CG}$ corresponds to the $5 \mathrm{hmC}$ feature vector. $\triangle 5 \mathrm{mCG} / \mathrm{CG} \& \Delta 5 \mathrm{hmCG} / \mathrm{CG}$ corresponds to concatenating $5 \mathrm{mCG}$ and $5 \mathrm{hmCG}$ feature vectors together for the classification. $\triangle 5 \mathrm{mCG} / \mathrm{CG}+\Delta 5 \mathrm{hmCG} / \mathrm{CG}$ corresponds to summing $5 \mathrm{mCG}$ and $5 \mathrm{hmCG}$ values before creating the feature vector for classification.

\section{Evaluation Framework}

ME-Class2 uses a random forest classifier which uses feature vectors from $5 \mathrm{mCG}, 5 \mathrm{hmCG}$ or both data to predict the direction of expression change. Random forests were built using 5001 trees. For the fetal to 6-week mouse brain comparison and dendritic cell analysis we used an intra-sample 10-fold cross validation. For the normal liver-lung and normal-tumor comparisons we performed cross-fold validation similar to that in Schlosberg et al. (13). In brief, we hold out each sample one by one for evaluation and 
then train on the remaining samples. To further minimize over-fitting, all genes from the validation sample are excluded from the samples used for training. For cortex-liver and cortex-lung comparisons, models were trained using 10-fold cross-validation, and all genes used for validation are excluded from the samples used for training.

\section{Unsupervised Clustering of 5hmCG and $5 \mathrm{mC}$}

Unsupervised hierarchical agglomerative clustering (complete linkage) was performed on $\triangle 5 \mathrm{mCG} / \mathrm{CG}$ and $\triangle 5 \mathrm{hmCG} / \mathrm{CG}$ in the region $[0,+2 \mathrm{~kb}]$ for the TSS for correctly predicted genes from ME-Class 2 . Subsetting our predictions required setting a working threshold for the probability of prediction. Therefore, we set the following range of probabilities of prediction for each experiment based on $90 \%$ accuracy at: [0.68, 1.0] fetal-6wk mouse, $[0.8,1.0]$ normal liver-tumor, $[0.7,1.0]$ normal-tumor liver and lung. Ranges were set at [0.8-1.0] for cortex-liver and cortex-lung based on 95\% accuracy due to the large number of genes accurately predicted for these samples. In the metagene plots of unsupervised results, $\Delta 5 \mathrm{mCG} / \mathrm{CG}+\Delta 5 \mathrm{hmCG} / \mathrm{CG}$ corresponds to the summation of $5 \mathrm{mCG}$ and $5 \mathrm{hmCG}$.

\section{RESULTS}

\section{Differential $5 \mathrm{hmCG}$ at promoter and promoter-proximal regions is more important than gene-body $5 \mathrm{hmCG}$ in predicting expression changes}

We extended ME-Class to simultaneously incorporate $5 \mathrm{mCG}$ and $5 \mathrm{hmCG}$ information from high resolution genomic data from WGBS, TAB-seq and oxBS-seq. We used the three best performing models for associating $5 \mathrm{mCG}$ and gene expression (13) to understand which model performed the best at the new task of using combined $5 \mathrm{mCG}$ and $5 \mathrm{hmCG}$ data (Fig. 1a). The first is a high-resolution promoter signature (HRPS) that interpolates a signature around the window $+/-5 \mathrm{~kb}$ of the TSS for both $5 \mathrm{mCG}$ and $5 \mathrm{hmCG}$ signals. We previously identified this model as optimal for associating $5 \mathrm{mCG}$ and expression changes (13). The second model, which we call "regions of interest" (ROI), bins methylation data upstream of the TSS and across gene features such as first and internal exons and introns (21). We further compared these methods to a whole-scaled gene (WSG) approach, which is based on a scaling method to compare whole gene signals across genes and is commonly used to capture correlations between gene body methylation and expression (8).

We initially benchmarked these models using a set of WGBS and TAB-seq data from fetal and 6-week mouse brains. To evaluate performance, we plot the accuracy versus 1 - reject rate for each model. This performance metric allows us to focus on only the genes with the highest quality predictions given some confidence threshold. The underlying premise is that only some genes should have associated DNA methylation and expression changes, not all. We demonstrate good performance to predict gene expression change as measured by both accuracy versus 1-reject rate (Fig. 1c) and ROC analysis (Fig. 1d) for all models. In the HRPS model we predict differential expression in 216 genes with greater than 
$90 \%$ accuracy, which outperforms ROI and WSG models for which we detect a similar number of genes, but at only $83 \%$ and $88 \%$ accuracy respectively. Both methods that capture the area around the TSS at high resolution (HRPS and WSG) out-perform other methods. Interestingly, models that incorporate features from the gene body (ROI and WSG) do not perform better than those that only model the data around the TSS at high resolution (HRPS). Further, direct addition of gene-body features to the HRPS model (HRPS+ROI) does not increase performance. Random forest feature importance analysis indicates that $5 \mathrm{mCG}$ and $5 \mathrm{hmCG}$ changes within $2 \mathrm{~kb}$ and primarily downstream of the TSS into the first intron are the most important regions for successful classification (Fig. 2).

\section{Addition of $5 \mathrm{hmCG}$ data improves ME-Class2 performance}

We next sought to determine whether models trained using both $5 \mathrm{hmCG}$ and $5 \mathrm{mcG}$ data outperformed those trained only using $5 \mathrm{mCG}$ data. Figure $3 \mathrm{a}-\mathrm{c}$ shows that including $5 \mathrm{mCG}$ and $5 \mathrm{hmCG}$ as independent features boosts ME-Class2 performance in the comparison of mouse fetal and 6-week brains and human cortex versus liver and lung using the HRPS model (corresponding ROC curves are in Supplementary Fig. S1). For mouse brain comparisons, the model using 5hmCG and 5mCG data predicted 112 genes at greater than $90 \%$ accuracy. Using $5 \mathrm{mCG}$ or $5 \mathrm{hmCG}$ data alone, the accuracy for a similar number of genes was only $82 \%$ and $75 \%$ respectively. Similar increases in performance with the inclusion of $5 \mathrm{hmCG}$ data were observed for other models including WSG, ROI, and HRPS + ROI (Supplementary Fig. S2). Using the HRPS model, $\triangle 5 \mathrm{mCG}+\Delta 5 \mathrm{hmC}$, which is effectively what is measured by only WGBS data in the absence of a $5 \mathrm{hmC}$-specific assay, performed equivalent to $\triangle 5 \mathrm{mCG}$ alone (Supplementary Fig. S3).

We also observe similar performance gains in human cortex vs liver and lung comparisons (Fig. 3). For cortex versus liver, differential expression of an average of 480 genes per sample $(493,493$, and 453 for each sample respectively) could be predicted at $90 \%$ accuracy using $5 \mathrm{hmCG}$ and $5 \mathrm{mCG}$ changes, but this accuracy fell to $82 \%$ and $77 \%$ for $5 \mathrm{mCG}$ and $5 \mathrm{hmCG}$ alone respectively. Meanwhile for cortex versus lung, differential expression of an average of 278 genes per sample (359, 266, and 209 for each sample respectively) could be predicted at $90 \%$ accuracy using $5 \mathrm{hmCG}$ and $5 \mathrm{mCG}$ changes, but this accuracy fell to $81 \%$ and $72 \%$ for $5 \mathrm{mCG}$ and $5 \mathrm{hmCG}$ alone respectively. We also observed an increase in performance comparing bacterially infected and non-infected dendritic cells, although the addition of $5 \mathrm{hmCG}$ data only allowed the prediction of differential expression for 16 total genes at greater than $93 \%$ accuracy (Fig. 3f, Supplementary Table S3). However, we do not observe such performance gains for all samples. We did not observe any substantial difference between $5 \mathrm{mCG}$ only and $5 \mathrm{mCG}$ and $5 \mathrm{hmCG}$ models in comparisons involving human lung and liver tissues across three individuals (Fig. 3d,g,h), or in normal-tumor comparisons from three lung and two liver tumors (Fig. 3e,g,h). Feature importance analysis of cortex vs liver, cortex vs lung, and infected dendritic cells all support the region within 2-3 kb of the TSS as most important for predicting expression change (Supplementary Fig. S4). 


\section{Predictive methylation signatures in non-brain tissues and tumors are solely dependent on changes in $5 \mathrm{mCG}$}

Similar unsupervised clustering of highly predictive liver-lung and cancer-specific genes show why the addition of $5 \mathrm{hmCG}$ data did not increase performance in these comparisons (Supplementary Fig. S5). The differential methylation signatures produced from these clusters in each case show that the net $\Delta 5 \mathrm{mCG}+\Delta 5 \mathrm{hmCG}$ levels closely follow the $\Delta 5 \mathrm{mCG}$ levels, with little difference in $5 \mathrm{hmCG}$ in all clusters. The observed 5mCG patterns in each cluster resemble those we previously found in other tissues(13) and cancer cell lines (22). This implies that promoter $5 \mathrm{mCG}$, rather than $5 \mathrm{hmCG}$, is primarily associated with gene expression change in cancer.

\section{ME-Class2 identifies $5 \mathrm{hmCG}$ and $5 \mathrm{mCG}$ signatures in brain tissues}

To better understand why we observed a boost in performance by including $5 \mathrm{hmCG}$ in brain and cortex comparisons, we conducted post-hoc unsupervised clustering analysis of identified signatures of $5 \mathrm{hmCG}$ and $5 \mathrm{mCG}$ that associate with expression change using the mouse fetal and 6-week brain comparison. We observe three distinct classes of differential 5hmCG and 5mCG signatures (Fig. 4a-c). In Figure 4a, we observe increases in both $5 \mathrm{mCG}$ and $5 \mathrm{hmCG} 3$ ' proximal to the TSS, which associate with a decrease in expression. This contrasts to the signature observed in cluster C2 (Fig. 4b). These genes also decrease in expression; however, while the 5mCG signal decreases 3' proximal to the TSS, the 5hmCG increases over the same region. There is no substantial change in the net $\Delta 5 \mathrm{mCG}+\Delta 5 \mathrm{hmCG}$ level, indicating that the primary feature in this cluster is a conversion from $5 \mathrm{mCG}$ to stable $5 \mathrm{hmCG}$ rather than demethylation. While most of the observed patterns cluster because of changes in $5 \mathrm{mC}$ data, observation of the $\mathrm{C} 2$ cluster is entirely dependent on the addition of $5 \mathrm{hmC}$ data. A third cluster (C3, Fig. 4c) comprises a set of genes that increase in expression and are again characterized by $5 \mathrm{mCG}$ decreases and $5 \mathrm{hmCG}$ increase 3 ' proximal to the TSS. In this case however, the net amount of $5 \mathrm{mCG}+5 \mathrm{hmCG}$ decreases indicating $5 \mathrm{hmCG}$ plays a role as an intermediate toward demethylation. Differential methylation signatures similar to those found in clusters $\mathrm{C} 1$ and $\mathrm{C} 3$ were also observed in human cortex versus liver and lung comparisons (Supplementary Fig. S6).

To better understand whether $5 \mathrm{hmCG}$ marked promoters associated with gene repression we examined expression levels of genes in each cluster across mouse development. Cluster $\mathrm{C} 2$ genes which are marked by $5 \mathrm{mCG}$ alone in the fetal cortex have much lower expression as a whole than genes that gain $5 \mathrm{mCG}$ and $5 \mathrm{hmCG}$ found in cluster $\mathrm{C} 1$ ( $p<0.009$, Wilcoxon test, Fig. 4d-f). This is in agreement with our finding that $5 \mathrm{hmCG}$ within $2 \mathrm{~kb}$ of the TSS associates with the repression of transcription. To test whether this conclusion would hold true in an alternative dataset, we first used feature importance analysis (Fig. 2a) to identify the region from [-800bp, 2100bp] around the TSS for both $5 \mathrm{mCG}$ and $5 \mathrm{hmCG}$ signals that contributes the greatest to classification in the fetal-6wk brain comparison. Next, we calculated the average $5 \mathrm{mC}, 5 \mathrm{hmC}$, and unmodified $\mathrm{C}$ content across this region for all genes in granule 
cells. Agreeing with our hypothesis, genes primarily marked with high levels of either $5 \mathrm{mCG}(\mathrm{p}<2 \mathrm{e}-16$, Bonferroni adjusted Wilcoxon test) or $5 \mathrm{hmCG}(\mathrm{p}<2 \mathrm{e}-7$, Bonferroni adjusted Wilcoxon test) are generally not expressed (Fig. 4g).

Lastly to understand whether the conversion or demethylation events are a potential cause of transcriptional change, we examined whether genes from each cluster identified in fetal-6wk comparison above were differentially expressed in Tet1-/- mouse cortex (10). Cluster C1 is characterized by predominantly increased $5 \mathrm{mCG}$ levels and thus, as expected, there was no significant difference in the expression of these genes after removal of Tet1. Genes in cluster C2 that were down-regulated in 6-week mouse brain, which had undergone a conversion of $5 \mathrm{mCG}$ to $5 \mathrm{hmCG}$ (with no net decrease of $5 \mathrm{mCG}+5 \mathrm{hmCG}$ ), were found to generally increase in expression in Tet1 -/- mouse cortex relative to WT $(p=0.037$, Bonferroni adjusted Wilcoxon test). Surprisingly, there was also no change in expression for genes undergoing Tet-mediated demethylation (cluster C3). This could be because $5 \mathrm{hmC}$-mediated demethylation occurs as a consequence of transcription. In agreement, transcription factor complexes have been implicated to recruit Tet1 leading to $5 \mathrm{hmC}$ mediated demethylation mediated by PPAR $\gamma$ in differentiated ES cells (23). However, our analysis has several limitations that could explain the lack of an observed effect. The promoters of selected genes that are differentially expressed in Tet1 -/- cortex and hippocampus were shown to increase in $5 \mathrm{mCG}$ levels by only $11-50 \%$, which may be insufficient for many genes to show a change in expression (10). Additionally, we cannot rule out that other Tet members play a compensatory role in the absence of Tet1. In support of the fact that the observed demethylation may activate transcription, a gene found in cluster C3, regulator of $G$ protein signaling RGS14, was shown previously to up-regulate after demethylation of neural progenitors using Dnmt inhibitors(24). In summary, while these data support a role for $5 \mathrm{hmCG}$ as a functional repressor, whether demethylation is a cause or consequence of transcriptional silencing or whether there is a context-dependent component is unclear.

\section{ME-Class2 identifies genes associated with neurodevelopmental disorders and neuronal development}

Gene ontology analysis using DAVID (25) revealed genes associated with neurodevelopmental disorders and basic neuronal development in all clusters (Supplementary Table S4). Several of these have been implicated to have differential methylation associated with different disorders including Shank2 in cluster C2 and Nrxn1, Pacsin1, and Grin1 in cluster C3. Shank2, a synaptic protein, has previously been shown to change methylation in the developing human brain and is associated with neurodevelopmental disorders (26). Methylation of Grin1, a component of NMDA receptor complexes, is associated with depression in children (27). Nrxn1 has previously been discovered as having a high ranking meQTL in 110 human hippocampus samples (28). Age-related DNA methylation changes have been found in Nrxn1, which has been implicated in schizophrenia and autism (29). Methylation of PACSIN1 is associated with substance-use risk (30). Importantly, our analysis suggests that $5 \mathrm{hmCG}$ may regulate disease-risk genes differently depending on whether it plays a role in repression or demethylation. 


\section{DISCUSSION}

We successfully extended ME-Class to predict gene expression classification from both $5 \mathrm{hmCG}$ and $5 \mathrm{mCG}$. Feature importance analysis shows that even in tissues with substantial $5 \mathrm{hmCG}, 5 \mathrm{mCG}$ is still the most useful mark for predicting expression changes (Fig. 2, Supplementary Fig. S4). 5hmCG models alone perform very poorly, which demonstrates the importance of considering $5 \mathrm{hmC}$ in the context of $5 \mathrm{mC}$ to understand potential associations and effects on transcription. Unsupervised analysis revealed a set of down-regulated genes with no net change in $5 \mathrm{mCG}+5 \mathrm{hmCG}$ levels, but for which $5 \mathrm{mCG}$ levels decrease and $5 \mathrm{hmCG}$ levels rise. Models using only WGBS data would miss these genes since WGBS only observes the net change in $5 \mathrm{mCG}+5 \mathrm{hmCG}$. For other tissues with minimal amounts of $5 \mathrm{hmCG}$ it is unlikely that obtaining TAB- or oxBS-seq data will provide more information over what is already found using WGBS (5mCG $+5 \mathrm{hmCG})$.

Our results suggest that the incorporation of $5 \mathrm{mCG}$ and $5 \mathrm{hmCG}$ marks in the gene body or outside a 2-3kb window from the TSS has little impact on the ability to associate methylation and transcription changes. The direct addition of gene body features based on differential methylation of internal exons and introns using the ROI approach led to no boost in performance. Feature importance analysis (Fig. 2, Supplementary Fig. S4) clearly indicates that for all models, the features within 2-3 kb of the TSS are most essential for prediction and that gene body features greater than 2-3 kb from the TSS are of limited utility. Taken together, this implies either that average gene body $5 \mathrm{hmCG}$ plays little functional role in the regulation of transcription, or that gene body information is redundant with that found within 2-3 kb of the promoter. Another alternative is that gene body $5 \mathrm{hmCG}$ plays a subtle effect on gene regulation that can only be uncovered with additional training data. For example, these models do not effectively incorporate individual regulatory elements such as enhancers or cryptic promoters found in the gene body that may have context-dependent contributions.

Our results further show that the addition of $5 \mathrm{hmCG}$ data has the greatest effect on performance in samples with substantial amounts of $5 \mathrm{hmCG}$ such as found in the brain. $5 \mathrm{hmCG}$ accumulates in the adult brain as can be observed in Fig. 4a, where $5 \mathrm{hmCG}$ increases in most regions around the TSS in 6wk relative to fetal mouse brain. Post-mitotic neurons have high $5 \mathrm{hmCG}$ levels and thus these samples benefit the most from inclusion of $5 \mathrm{hmCG}$ for predictions. $5 \mathrm{hmCG}$ exists at relatively low levels in liver (2.27\%-5.68\%) and lung (1.94-3.04\%) (12) in comparison to mouse brain (17.2\%) (7) and human cortex $(13 \%)(16)$ tissue. $5 \mathrm{hmCG}$ is an intermediate cytosine modification which is not replicated during mitosis. Lack of gene expression correlating $5 \mathrm{hmCG}$ patterns in normal lung and liver may be because dividing cells in these tissues passively dilute $5 \mathrm{hmCG}$ from their genomes. Thus, the scarcity of $5 \mathrm{hmCG}$ might explain its lack of predictive ability for expression class change in non-neuronal tissues. In agreement, clustering analysis of $5 \mathrm{mCG}$ and $5 \mathrm{hmCG}$ signals of predictive genes did not reveal a cluster of $5 \mathrm{mCG}$ to $5 \mathrm{hmCG}$ conversion as we observed in the model of mouse brain development. Instead, the patterns of differential $5 \mathrm{hmCG}$ and $5 \mathrm{mCG}$ closely follows that of $5 \mathrm{mCG}$ alone across all predictive signatures. 
However, we cannot rule out that 5hmCG inclusion in tissues with low amounts of $5 \mathrm{hmCG}$ might facilitate the identification of a few rare genes regulated by $5 \mathrm{hmCG}$, which cannot be assessed with the limited amount of training data currently available.

Lastly our work points to a potential new mechanism of $5 \mathrm{hmCG}$ mediated repression of gene promoters independent of that observed by $5 \mathrm{mCG}$. We identify that conversion of $5 \mathrm{mCG}$ to $5 \mathrm{hmCG}$ primarily is associated with the downregulation of gene expression and many of these genes are upregulated upon the removal of Tet 1 . Since there have been very few proteins identified that specifically bind $5 \mathrm{hmC}$ relative to $5 \mathrm{mC}(4)$, it is possible that $5 \mathrm{hmCG}$-associated gene silencing could instead be caused by Tet1-recruitment of interacting partners, such as Sin3A and OGT, which have been shown to be involved in Tet1-dependent silencing of LINE-1 (31). It is further unclear at this point why 5hmCG would stabilize in some genes versus other, and complicating matters is that how much active versus passive demethylation occurs via $5 \mathrm{hmCG}$ is still a point of contention. It may be that demethylation is the dominant mechanism prior to neurons exiting the cell cycle, while stable $5 \mathrm{hmCG}$ occurs after.

ME-Class2 demonstrates that incorporating $5 \mathrm{hmCG}$ information is critical for prediction of gene expression changes in samples with high levels of $5 \mathrm{hmC}$ such as the brain and neurons. ME-Class 2 has identified a class $5 \mathrm{mCG} / 5 \mathrm{hmCG}$ patterns that show the conversion from $5 \mathrm{mCG}$ to $5 \mathrm{hmCG}$ in the 3 ' proximal region of the promoter in a model of mouse brain development. We speculate that these patterns of $5 \mathrm{mCG}$ and $5 \mathrm{hmCG}$ coordinate with additional silencing factors potentially recruited either directly by $5 \mathrm{hmCG}$ or by the Tet enzymes in a context-specific manner. As the field continues to collect genome-wide, differential DNA methylation (including both $5 \mathrm{mC}$ and $5 \mathrm{hmC}$ ), tools such as ME-Class2 will prove invaluable for the interpretation of this epigenomic data and will guide mechanistic studies into the integrated function of $5 \mathrm{mC}$ and $5 \mathrm{hmC}$ in human disease.

\section{AVAILABILITY}

ME-Class2 is an open source collaborative initiative available in the GitHub repository (https://github.com/jredwards417/me-class2)

\section{ACKNOWLEDGEMENT}

We would also like to thank Jerry Fong and Scot Matkovich for critical reading of the manuscript, and Kilian Weinberger and Harrison Gabel for helpful discussions.

\section{FUNDING}

This work was supported by National Institutes of Health [grant number R01 GM108811 to JRE]. Further support for CES was from the National Institutes of Health Genome Analysis Training Program [ grant number T32 HG000045]. 


\section{REFERENCES}

1. Ito,S., Shen,L., Dai,Q., Wu,S.C., Collins,L.B., Swenberg,J.A., He,C. and Zhang,Y. (2011) Tet proteins can convert 5-methylcytosine to 5-formylcytosine and 5-carboxylcytosine. Science, 333, 1300-1303.

2. Branco,M.R., Ficz,G. and Reik,W. (2012) Uncovering the role of 5-hydroxymethylcytosine in the epigenome. Nat Rev Genet, 13, 7-13.

3. Raiber,E.-A., Murat,P., Chirgadze,D.Y., Beraldi,D., Ben F Luisi and Balasubramanian,S. (2015) 5Formylcytosine alters the structure of the DNA double helix. Nat Struct Mol Biol, 22, 44-49.

4. Iurlaro,M., Ficz,G., Oxley,D., Raiber,E.-A., Bachman,M., Booth,M.J., Andrews,S., Balasubramanian,S. and Reik,W. (2013) A screen for hydroxymethylcytosine and formylcytosine binding proteins suggests functions in transcription and chromatin regulation. Genome Biol, 14, R119.

5. Edwards,J.R., Yarychkivska,O., Boulard,M. and Bestor,T.H. (2017) DNA methylation and DNA methyltransferases. Epigenetics Chromatin, 10, 23.

6. Kohli,R.M. and Zhang,Y. (2013) TET enzymes, TDG and the dynamics of DNA demethylation. Nature, 502, 472-479.

7. Lister,R., Mukamel,E.A., Nery,J.R., Urich,M., Puddifoot,C.A., Johnson,N.D., Lucero,J., Huang,Y., Dwork,A.J., Schultz,M.D., et al. (2013) Global epigenomic reconfiguration during mammalian brain development. Science, 341, 1237905-1237905.

8. Lister,R., Mukamel,E.A., Nery,J.R., Urich,M., Puddifoot,C.A., Johnson,N.D., Lucero,J., Huang,Y., Dwork,A.J., Schultz,M.D., et al. (2013) Global Epigenomic Reconfiguration During Mammalian Brain Development. Science (New York, N.Y.), 341, 1237905-627.

9. Dawlaty,M.M., Breiling,A., Le,T., Barrasa,M.I., Raddatz,G., Gao,Q., Powell,B.E., Cheng,A.W., Faull,K.F., Lyko,F., et al. (2014) Loss of Tet enzymes compromises proper differentiation of embryonic stem cells. Dev. Cell, 29, 102-111.

10. Rudenko,A., Dawlaty,M.M., Seo,J., Cheng,A.W., Meng,J., Le,T., Faull,K.F., Jaenisch,R. and Tsai,L.$\mathrm{H}$. (2013) Tet1 is critical for neuronal activity-regulated gene expression and memory extinction. Neuron, 79, 1109-1122.

11. Hon,G.C., Song,C.-X., Du,T., Jin,F., Selvaraj,S., Lee,A.Y., Yen,C.-A., Ye,Z., Mao,S.-Q., Wang,B.-A., et al. (2014) $5 \mathrm{mC}$ oxidation by Tet2 modulates enhancer activity and timing of transcriptome reprogramming during differentiation. Mol Cell, 56, 286-297.

12. Li,X., Liu,Y., Salz,T., Hansen,K.D. and Feinberg,A. (2016) Whole-genome analysis of the methylome and hydroxymethylome in normal and malignant lung and liver. Genome Res, 26, 1730-1741.

13. Schlosberg,C.E., Vanderkraats,N.D. and Edwards,J.R. (2017) Modeling complex patterns of differential DNA methylation that associate with gene expression changes. Nucleic Acids Res, 45, 5100-5111.

14. Pacis,A., Tailleux,L., Morin,A.M., Lambourne,J., Maclsaac,J.L., Yotova,V., Dumaine,A., Danckaert,A., Luca,F., Grenier,J.-C., et al. (2015) Bacterial infection remodels the DNA methylation landscape of human dendritic cells. - PubMed - NCBI. Genome Res, 25, 1801-1811.

15. Mellén,M., Ayata,P. and Heintz,N. (2017) 5-hydroxymethylcytosine accumulation in postmitotic neurons results in functional demethylation of expressed genes. Proceedings of the National Academy of Sciences of the United States of America, 114, E7812-E7821. 
16. Wen,L., Li,X., Yan,L., Tan,Y., Li,R., Zhao,Y., Wang,Y., Xie,J., Zhang,Y., Song,C., et al. (2014) Wholegenome analysis of 5-hydroxymethylcytosine and 5-methylcytosine at base resolution in the human brain. Genome Biol, 15, R49.

17. Brawand,D., Soumillon,M., Necsulea,A., Julien,P., Csárdi,G., Harrigan,P., Weier,M., Liechti,A., Aximu-Petri,A., Kircher,M., et al. (2011) The evolution of gene expression levels in mammalian organs. Nature, 478, 343-348.

18. Qu,J., Zhou,M., Song,Q., Hong,E.E. and Smith,A.D. (2013) MLML: consistent simultaneous estimates of DNA methylation and hydroxymethylation. Bioinformatics, 29, 2645-2646.

19. Kim,D., Ben Langmead and Salzberg,S.L. (2015) HISAT: a fast spliced aligner with low memory requirements. Nat Methods, 12, 357-360.

20. Liao,Y., Smyth,G.K. and Shi,W. (2014) featureCounts: an efficient general purpose program for assigning sequence reads to genomic features. Bioinformatics, 30, 923-930.

21. Lou,S., Lee,H.-M., Qin,H., Li,J.-W., Gao,Z., Liu,X., Chan,L.L., KI Lam,V., So,W.-Y., Wang,Y., et al. (2014) Whole-genome bisulfite sequencing of multiple individuals reveals complementary roles of promoter and gene body methylation in transcriptional regulation. Genome Biol, 15, 408.

22. Vanderkraats,N.D., Hiken,J.F., Decker,K.F. and Edwards,J.R. (2013) Discovering high-resolution patterns of differential DNA methylation that correlate with gene expression changes. Nucleic Acids Res, 41, 6816-6827.

23. Fujiki,K., Shinoda,A., Kano,F., Sato,R., Shirahige,K. and Murata,M. (2013) PPARy-induced PARylation promotes local DNA demethylation by production of 5-hydroxymethylcytosine. Nat Commun, 4, 2262.

24. Tuggle,K., Ali,M.W., Salazar,H. and Hooks,S.B. (2014) Regulator of G Protein Signaling Transcript Expression in Human Neural Progenitor Differentiation: R7 Subfamily Regulation by DNA Methylation. Neurosignals, 22, 43-51.

25. Huang,D.W., Sherman,B.T. and Lempicki,R.A. (2009) Systematic and integrative analysis of large gene lists using DAVID bioinformatics resources. Nat Protoc, 4, 44-57.

26. Spiers,H., Hannon,E., Schalkwyk,L.C., Smith,R., Wong,C.C.Y., O'Donovan,M.C., Bray,N.J. and Mill,J. (2015) Methylomic trajectories across human fetal brain development. Genome Res, 25, 338-352.

27. Weder,N., Zhang,H., Jensen,K., Yang,B.Z., Simen,A., Jackowski,A., Lipschitz,D., DouglasPalumberi,H., Ge,M., Perepletchikova,F., et al. (2014) Child Abuse, Depression, and Methylation in Genes Involved With Stress, Neural Plasticity, and Brain Circuitry. Journal of the American Academy of Child \& Adolescent Psychiatry, 53, 417-424.e5.

28. Schulz,H., Ruppert,A.-K., Herms,S., Wolf,C., Mirza-Schreiber,N., Stegle,O., Czamara,D., Forstner,A.J., Sivalingam,S., Schoch,S., et al. (2017) Genome-wide mapping of genetic determinants influencing DNA methylation and gene expression in human hippocampus. Nat Commun, 8, 1511.

29. Numata,S., Ye,T., Hyde,T.M., Guitart-Navarro,X., Tao,R., Wininger,M., Colantuoni,C., Weinberger,D.R., Kleinman,J.E. and Lipska,B.K. (2012) DNA Methylation Signatures in Development and Aging of the Human Prefrontal Cortex. The American Journal of Human Genetics, 90, 260-272.

30. Cecil,C.A.M., Walton,E., Smith,R.G., Viding,E., McCrory,E.J., Relton,C.L., Suderman,M., Pingault,J.B., McArdle,W., Gaunt,T.R., et al. (2016) DNA methylation and substance-use risk: a prospective, genome-wide study spanning gestation to adolescence. Transl Psychiatry, 6, e976-e976. 
bioRxiv preprint doi: https://doi.org/10.1101/318808; this version posted May 10, 2018. The copyright holder for this preprint (which was not certified by peer review) is the author/funder. All rights reserved. No reuse allowed without permission.

31. la Rica,de,L., Deniz,Ö., Cheng,K.C.L., Todd,C.D., Cruz,C., Houseley,J. and Branco,M.R. (2016) TETdependent regulation of retrotransposable elements in mouse embryonic stem cells. Genome Biol, $17,234$. 


\section{FIGURES}

a.

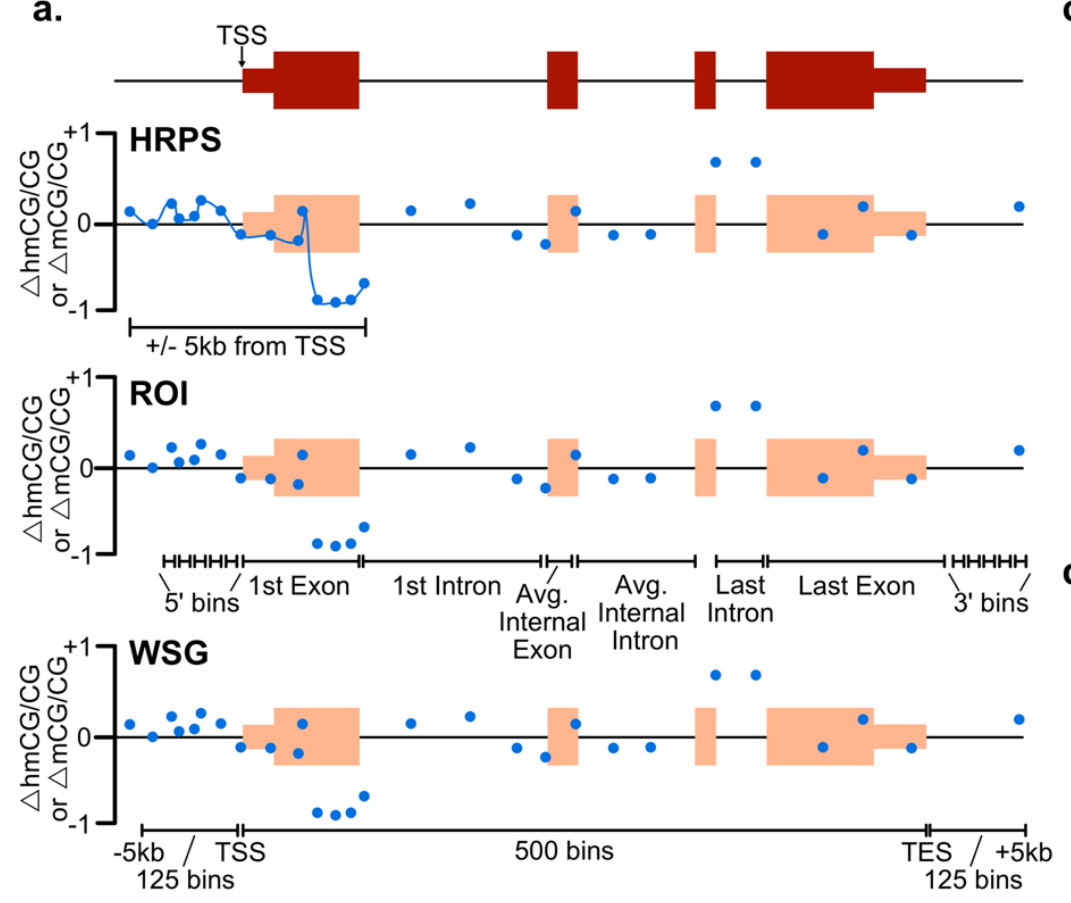

b.

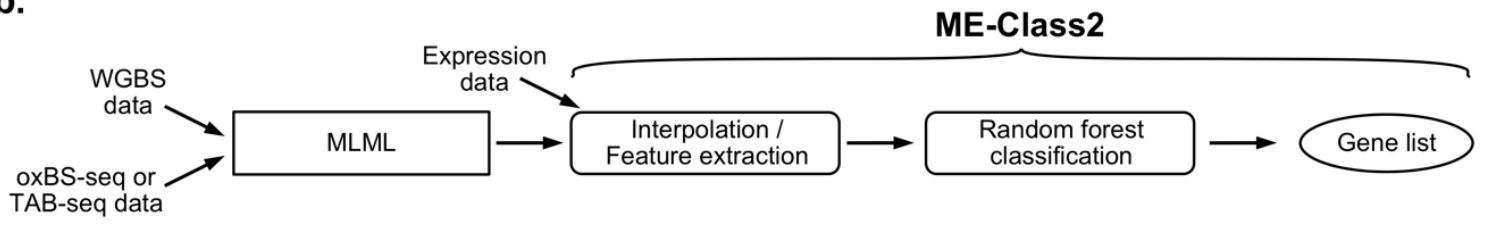

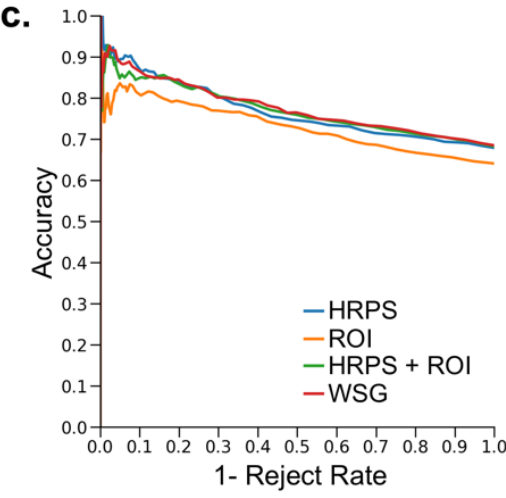

d.

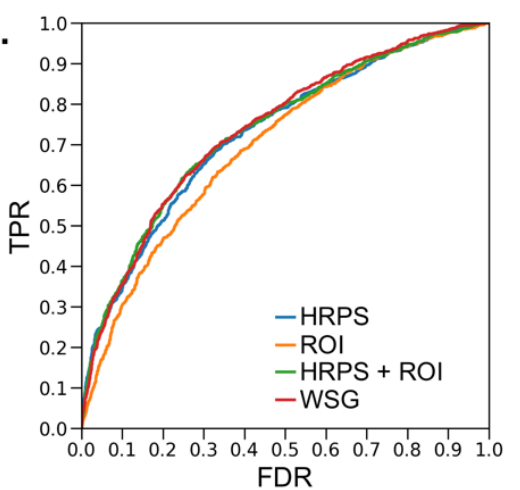

Figure. 1. (a) Cartoon example showing different models to encode methylation features for a gene for ME-Class2 analysis. $\triangle 5 \mathrm{mCG} / \mathrm{CG}$ and $\triangle 5 \mathrm{hmCG} / \mathrm{CG}$ refer to the differences between two samples. HRPS is high-resolution promoter signature; ROI is region of interest; WSG is whole-scale gene. TSS is transcription start site. Blue dots show example differential methylation (5hmCG or 5mCG). (b) MEClass2 workflow. (c,d) Performance of different gene models using ME-Class2 5mCG and 5hmCG data from fetal and 6-week mouse brain as evaluated using accuracy versus 1-reject rate (c) and ROC (receiver operating characteristic) curve analysis (d). 1 - reject rate is the fraction of genes with predicted associations between methylation and expression. ROC AUC are HRPS: 0.727 , HRPS + ROI: 0.735, WSG: 0.739, ROI: 0.699. 

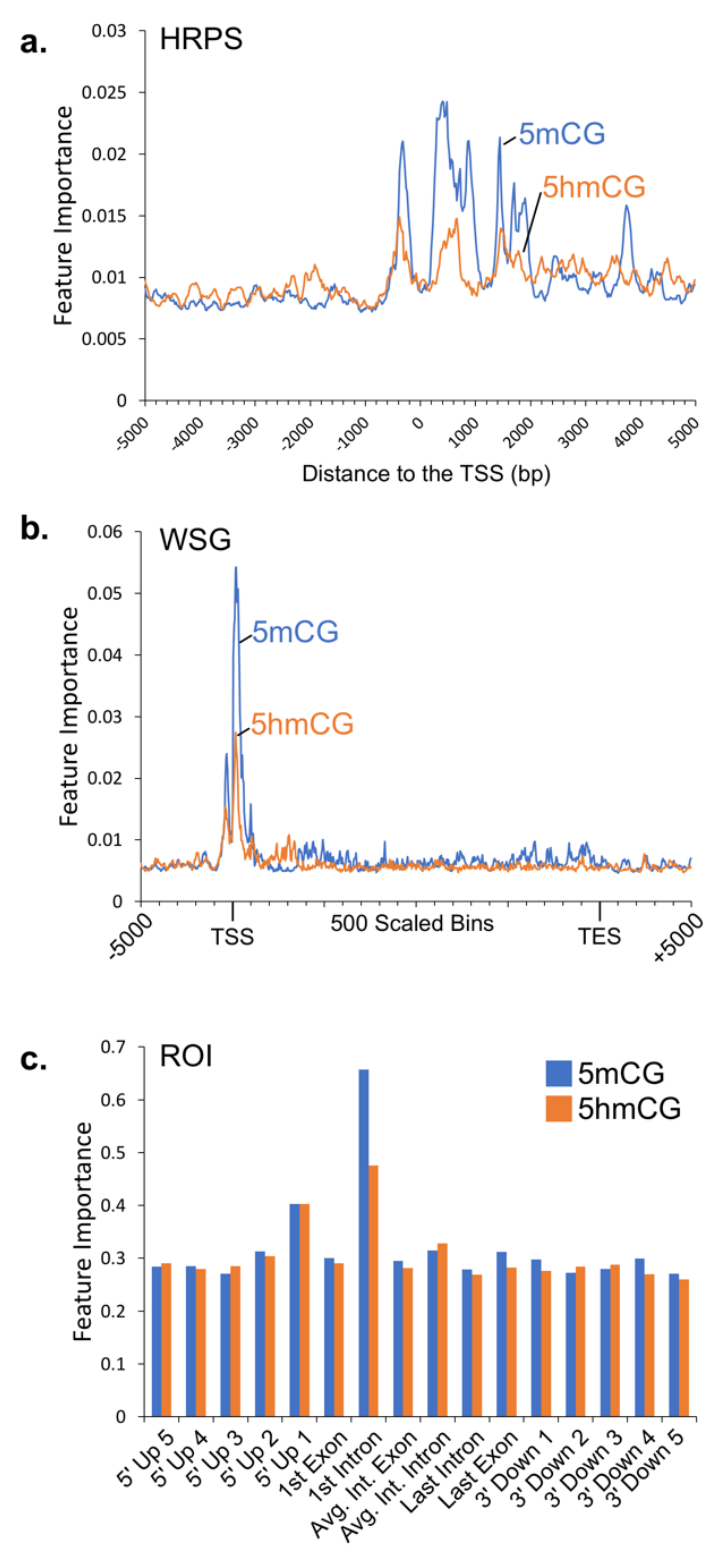

Figure 2. Feature importance for the ME-Class2 random forest classifier for fetal-6wk mouse brain $5 \mathrm{mCG}$ and $5 \mathrm{hmCG}$ data for (a) HRPS, (b) WSG, and (c) ROI data representations. 
a.

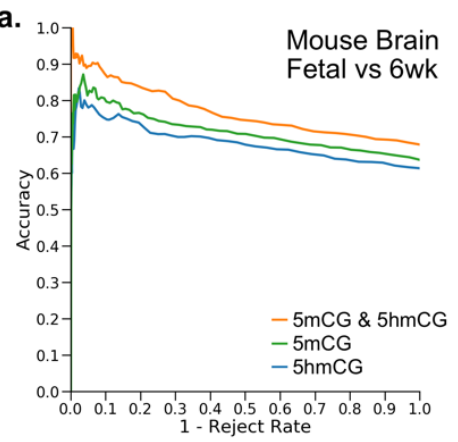

d.

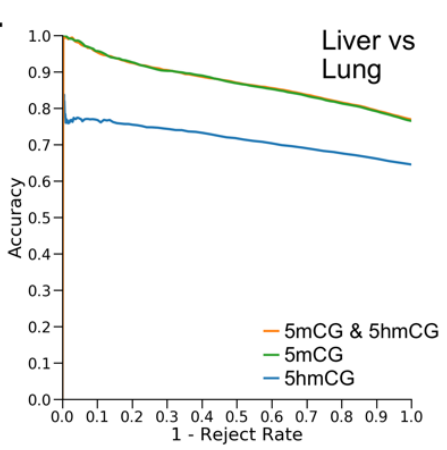

g. Accuracy vs 1-Reject Rate

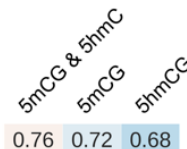

$\begin{array}{llll}\text { Mouse Brain } & 0.76 & 0.72 & 0.68\end{array}$

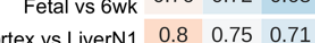

\begin{tabular}{ll|l|l|l} 
Cortex vs LiverN2 & 0.79 & 0.74 & 0.69
\end{tabular}

Cortex vs LiverN3 $0.74 \quad 0.67 \quad 0.64$

\begin{tabular}{llll} 
Cortex vs LungN1 & 0.77 & 0.7 & 0.61 \\
\hline
\end{tabular}

$\begin{array}{llll}\text { Cortex vs LungN2 } & 0.79 & 0.73 & 0.66\end{array}$

Cortex vs LungN3 $0.77 \quad 0.72 \quad 0.66$

$\begin{array}{llll}\text { LiverN1 vs LungN1 } & 0.9 & 0.9 & 0.78\end{array}$

LiverN1 vs LungN2 $0.91 \quad 0.91 \quad 0.79$

$\begin{array}{lllll}\text { LiverN1 vs LungN3 } & 0.91 & 0.9 & 0.82\end{array}$

$\begin{array}{lllll}\text { LiverN2 vs LungN1 } & 0.88 & 0.87 & 0.76\end{array}$

\begin{tabular}{ll|l|l|l} 
LiverN2 vs LungN2 & 0.88 & 0.88 & 0.76
\end{tabular}

\begin{tabular}{lll|l|l} 
LiverN2 vs LungN3 & 0.88 & 0.87 & 0.74
\end{tabular}

$\begin{array}{lllll}\text { LiverN3 vs LungN1 } & 0.84 & 0.83 & 0.63\end{array}$

$\begin{array}{lllll}\text { LiverN3 vs LungN2 } & 0.82 & 0.83 & 0.62\end{array}$

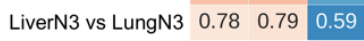

\begin{tabular}{ll|l|l|l|l} 
Liver Norm. vs Tumor 1 & 0.79 & 0.79 & 0.68
\end{tabular}

\begin{tabular}{ll|l|l|l|} 
Liver Norm. vs Tumor 2 & 0.88 & 0.86 & 0.6 \\
\hline
\end{tabular}

Lung Norm. vs Tumor $1 \quad 0.65 \quad 0.66 \quad 0.5$

\begin{tabular}{ll|l|l} 
Lung Norm. vs Tumor 2 & 0.52 & 0.52 & 0.53
\end{tabular}

Lung Norm. vs Tumor $3 \quad 0.76 \quad 0.76 \quad 0.61$

$\begin{array}{llll}\begin{array}{r}\text { Dendritic Cell } \\ \text { Inf vs Ctr }\end{array} & 0.64 & 0.61 & 0.63\end{array}$ b.

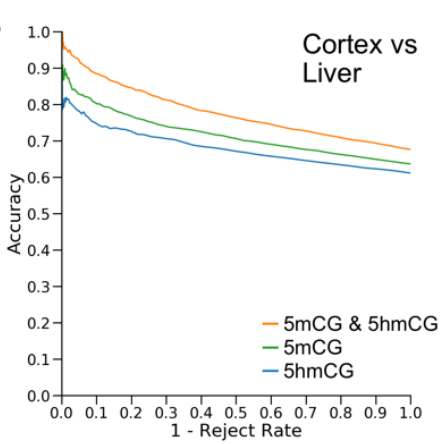

e.

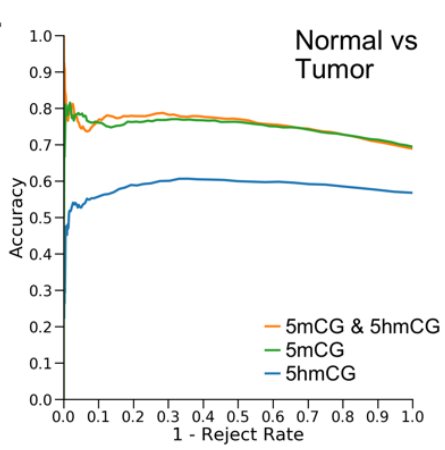

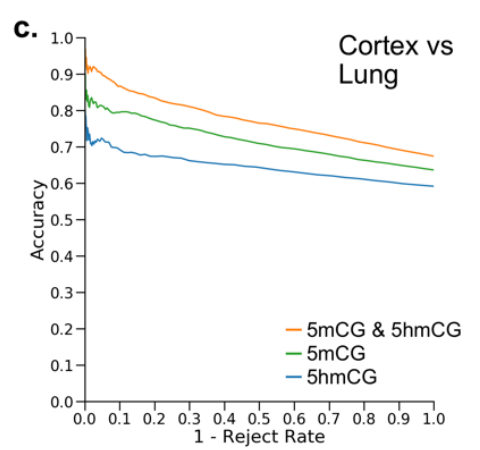

f.

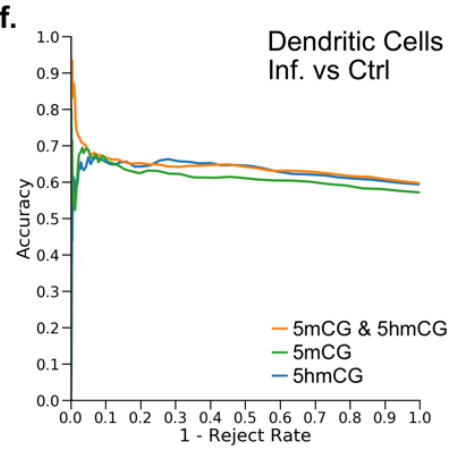

h. ROC

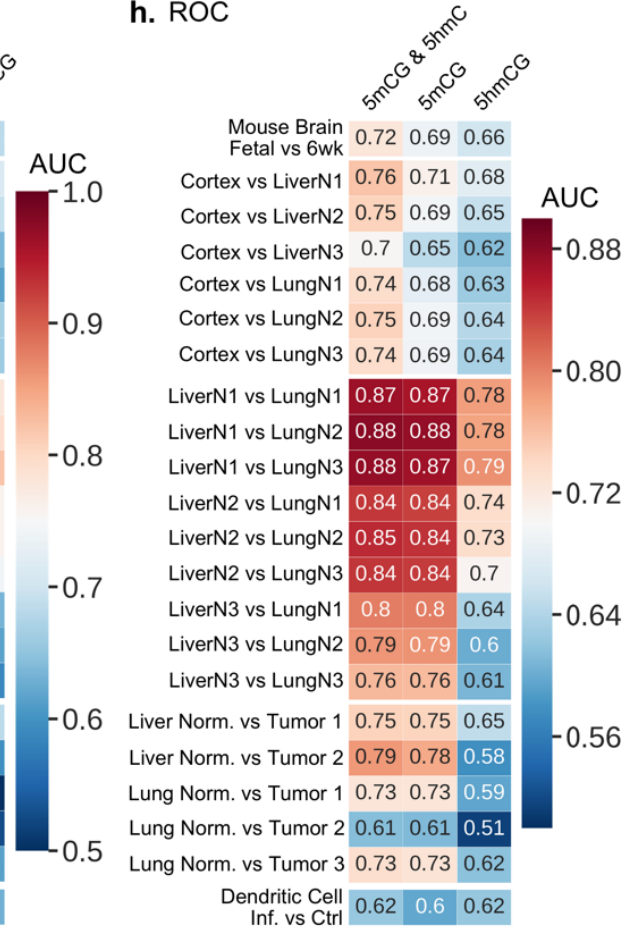

Figure. 3. (a-f) ME-Class2 performance (accuracy versus 1-reject rate) for different $5 \mathrm{mCG}$ and $5 \mathrm{hmCG}$ datasets using the HRPS feature model. The $5 \mathrm{mCG}$ and $5 \mathrm{mCG} \& 5 \mathrm{hmCG}$ curves directly overlap in panel d. Corresponding detailed ROC (receiver operating characteristic) curves are in Supplementary Fig. S2. (g) Area under the curve (AUC) for the accuracy vs 1-reject rate curves and (h) ROC AUC for each individual sample comparison used in a-f. 


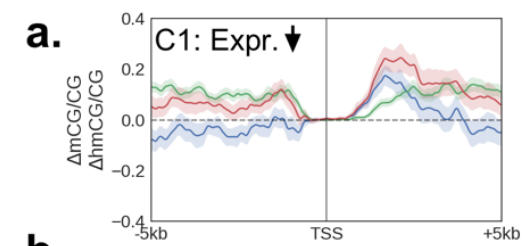

b.

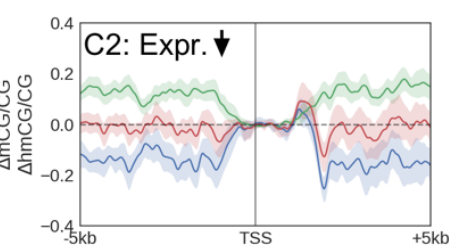

C.

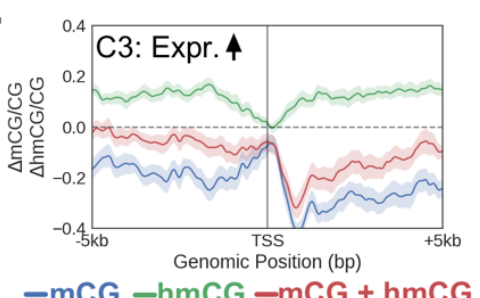

g.

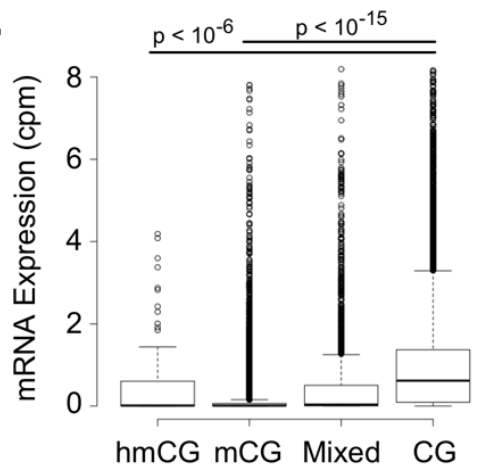

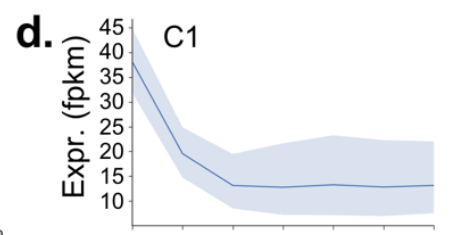

e.

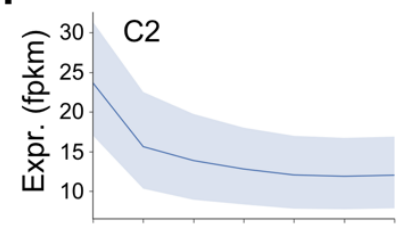

f.

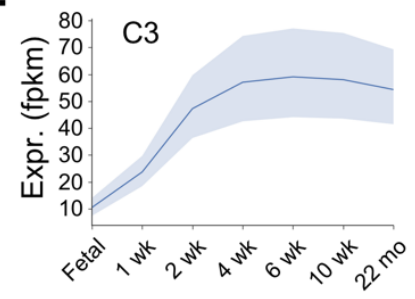

h.

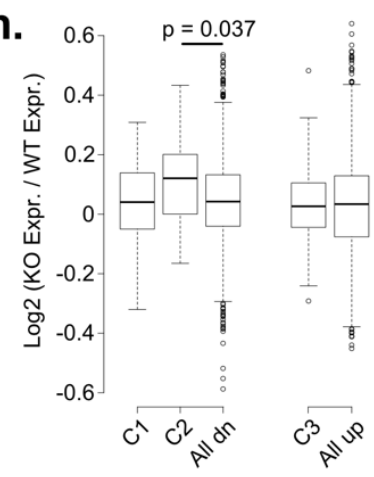

Figure 4. (a-c) Metagene plots for clusters of similar differential methylation signatures (6 wk - fetal) that are predictive of expression in the fetal-6wk mouse brain comparison. Shading indicates the $68 \%$ bootstrapped confidence interval. Cluster $\mathrm{C} 1: \mathrm{n}=76, \mathrm{C} 2: \mathrm{n}=29$, and C3: $n=70$. (d-f) Average expression of all genes found in C1, C2, and C3 clusters across mouse brain development. Shading indicates the 95\% confidence interval. (g) mRNA expression in granule cells of genes whose promoters (defined as [-800bp, $+2 \mathrm{~kb}$ ] around the TSS) are greater than $50 \%$ marked by $\mathrm{mCG}, \mathrm{hmCG}$, a combination of $\mathrm{mCG}$ and $\mathrm{hmCG}$, or CG (unmethylated). Outliers have been cropped for clarity. The original plot can be found in Supplementary Fig. S7. (h) Log2 expression changes in cortex from Tet1-/- mice versus cortex from WT mouse. All dn and all up correspond to all down- and up-regulated genes, respectively, in 6wk compared to fetal mouse brain. P-values computed using a Bonferroni adjusted Wilcoxon test. 